\title{
Navier-Stokes Airfoil Computations with Automatic Transition Prediction using the DLR TAU Code - A Sensitivity Study
}

\author{
A. KRUMBEIN \\ DLR, Institute of Aerodynamics and Flow Technology \\ Lilienthalplatz 7, 38108 Braunschweig, Germany, andreas.krumbein@dlr.de
}

\section{Summary}

The hybrid DLR RANS solver TAU coupled to a transition prediction module was successfully applied to a single-element airfoil automatically taking into account the locations of laminar-turbulent transition. The experimentally measured transition locations could be reproduced with very high accuracy. A sensitivity study of the parameters of the coupling procedure was performed in order to investigate the behaviour of the coupled system with respect to the accuracy and robustness of the iteration procedure for the transition locations. The transition prediction coupling structure and the underlying algorithm are described. The functions of the coupling parameters and their impact on the transition location iteration and the convergence of the simulations are described and documented.

\section{Introduction}

The modeling of laminar-turbulent transition in Reynolds-averaged Navier-Stokes (RANS) solvers is a necessary requirement for the computation of flows over airfoils and wings in the aerospace industry because it is not possible to obtain quantitatively correct results if the laminar-turbulent transition is not taken into account. For the design process of wings in industry, there exists the demand for a RANS-based computational fluid dynamics tool that is able to handle flows with laminar-turbulent transition automatically and autonomously. The first steps towards the setup of such a tool were made e.g. in [11], where a structured RANS solver and an $\mathrm{e}^{N}$-method, [12] and [18], were applied, and in [15], where a RANS solver, a laminar boundary-layer method [2] and an $\mathrm{e}^{N}$-method were coupled. There the boundary-layer method was used to produce highly accurate laminar, viscous layer data to be analysed by a linear stability code. The use of an $\mathrm{e}^{N}$-database method [14] results in a coupled program system that is able to handle transition prediction automatically. After the block-structured DLR RANS solver FLOWer [1] is in a well-engineered state with regard to automatic transition prediction and transitional flow modelling, [5-6] and [8], the DLR hybrid RANS solver TAU [3] was extended in a similar way in order to combine the benefit of a hybrid RANS code with an automatic transition prediction capability, [9-10]. In contrast to [9], where different strategies for the iteration of the transition locations were applied, in the present paper the established algorithm from [5-6] and [8] - taking into account the laminar separation points in the RANS computational grid - is applied. Additionally, physical models for transitional flow regions are introduced. Finally, a setting of the coupling parameters is specified which allows for a fast and accurate iteration of the transition locations. 


\section{Coupling of the TAU Code and the Transition Prediction Module}

The transition prediction module consists of a laminar boundary-layer code for swept, tapered wings [2], and an $\mathrm{e}^{N}$-database method for Tollmien-Schlichting (TS) instabilities [14]. The coupled system can be run in two different modes. Either the TAU code communicates the surface pressure distribution of the configuration to the laminar boundary-layer method, the laminar boundary-layer method computes all of the boundary-layer parameters that are needed for the $\mathrm{e}^{N}$-database method and the $\mathrm{e}^{N}$-database method determines new transition locations that are given back to the RANS solver ( $I^{s t}$ mode). Or the TAU code computes the boundary-layer parameters $\delta, \mathrm{H}_{\mathrm{i}}$ and $\mathrm{Re}_{\delta}{ }^{*}$ internally and communicates them directly to the $\mathrm{e}^{N}$-database method $\left(2^{\text {nd }}\right.$ mode). In [9-10] the influence of the cell number in wall normal direction and a comparison with results from a boundary-layer code are shown.

This coupled structure results in an iteration procedure for the transition locations within the iterations of the RANS equations. During the solution process of the RANS equations, the transition prediction module is called after a certain number of iterations, $\mathrm{k}_{\mathrm{cyc}}$, of the RANS iteration process. With the call of the module the solution process is interrupted and the module analyses the laminar boundary layers of both sides of an airfoil configuration. The determined transition locations, $x_{j}{ }^{\mathrm{T}}\left(\right.$ cycle $\left.=k_{\text {cyc }}\right)$ with $j=1, \ldots, n_{\text {loc }}$, where $n_{\text {loc }}$ is the number of transition points, are communicated to the RANS solver, which continues the solution process of the RANS equations. In so doing, the determination of the transition locations becomes an iteration process itself. The structure of the approach is outlined graphically in Fig. 1. At every call of the module the surface pressure, $\mathrm{c}_{\mathrm{p}}\left(\mathrm{cycle}=\mathrm{k}_{\mathrm{cyc}}\right)$, or the internally computed boundary-layer parameters along the surface of an airfoil are used as input for the transition prediction module. The viscous data is then subsequently analysed by the $\mathrm{e}^{N}$-database method. The algorithm for the transition prediction iteration works as follows:

a) The RANS solver is started as if a computation with prescribed transition locations should be performed. At this moment, the transition locations are set very far downstream on the upper and lower sides of the airfoil, e.g., at the trailing edge. The RANS solver now computes a fully laminar flow over the airfoil.

b) During the solution process of the RANS equations the laminar flow is checked for laminar separation points by the RANS solver. In the case that laminar separation is detected, the separation point is used as an approximation of the transition location and the computation is continued.

c) The RANS equations are iterated until the lift coefficient $c_{1}=c_{1}$ (cycles) has become constant with respect to the iteration cycles.

d) The transition prediction module is called. The $\mathrm{e}^{N}$-database method determines the transition locations on upper and lower sides of the airfoil. The procedure acts differently in the $1^{s t}$ mode and in the $2^{\text {nd }}$ mode. In the case that the $\mathrm{e}^{N}$-database method does not detect a transition location due to TS waves, two possibilities are implemented: Either the laminar separation point from the boundary-layer code is used as an approximation of the transition point $\left(1^{s t}\right.$ mode $)$ or the previous 
transition locations is kept unchanged ( $2^{\text {nd }}$ mode).

e) The current coordinate $x_{j}^{\mathrm{T}}\left(\right.$ cycle $\left.=\mathrm{k}_{\text {cyc }}\right)$, which is used as transition location, is underrelaxed. That is, as new transition locations the coordinates $\mathrm{x}_{\mathrm{j}}{ }^{\mathrm{T}}($ cycle $=$ $\mathrm{k}_{\mathrm{cyc}}$ ), which are located downstream of the coordinates $\mathrm{x}_{\mathrm{j}}^{\mathrm{T}}$, are used according to,

$$
\mathrm{x}_{\mathrm{j}}{ }^{* \mathrm{~T}}\left(\mathrm{k}_{\mathrm{cyc}}\right)=\mathrm{C}_{\mathrm{j}}^{\mathrm{T}}\left(\mathrm{k}_{\mathrm{cyc}}\right) \mathrm{x}_{\mathrm{j}}^{\mathrm{T}}\left(\mathrm{k}_{\mathrm{cyc}}\right) \quad \text { with } \mathrm{j}=1, \ldots, \mathrm{n}_{\mathrm{loc}},
$$

with $C_{j}{ }^{T}\left(k_{c y c}\right)>1$. The underrelaxation of the determined transition locations prevents the case that at an unconverged stage during the transition location iteration transition coordinates which are determined too far upstream might not be shifted downstream again.

f) As convergence criterion $\Delta \mathrm{x}_{\mathrm{j}}{ }^{\mathrm{T}, 1}<\varepsilon \approx 1 \%$ with $\Delta \mathrm{x}_{\mathrm{j}}{ }^{\mathrm{T}, 1}=\mid \mathrm{x}_{\mathrm{j}}{ }^{\mathrm{T}}\left(\mathrm{k}_{\mathrm{cyc}}^{1}\right)-\mathrm{x}_{\mathrm{j}}{ }^{* \mathrm{~T}}\left(\mathrm{k}^{1-}\right.$ $\left({ }_{\text {cyc }}\right) \mid$ is applied, where 1 is the current iteration step. In the case that the criterion is satisfied the iteration for $\mathrm{x}_{\mathrm{j}}^{\mathrm{T}}$ is finished, else the algorithm loops back to station $\mathrm{b}$ ).

\section{Generation of Transitional Flow Regions}

In the case that a new transition location has been determined, the laminar, transitional and turbulent flow regions must be generated within the computational grid. The generation of the different regions is done by setting a real value flag $\mathrm{FLG}_{\mathrm{lt}}$ at each point in the computational grid that is applied to the value of the source term $\mathrm{S}_{\mathrm{tp}}$ of the turbulence production, which is computed for every point in the flow field. $\mathrm{FLG}_{\mathrm{lt}}$ is applied in the following way to all of the points of the computational grid,

$$
\mathrm{S}_{\mathrm{tp}}{ }^{\text {code }}\left(\mathrm{P}_{\mathrm{F}}\right)=\mathrm{S}_{\mathrm{tp}}\left(\mathrm{P}_{\mathrm{F}}\right) * \operatorname{MIN}\left[\mathrm{FLG}_{\mathrm{lt}}\left(\mathrm{P}_{\mathrm{F}}\right), 1\right]
$$

with $\mathrm{FLG}_{\mathrm{lt}}\left(\mathrm{P}_{\mathrm{F}}\right)=0.0$ for a laminar surface point, $\mathrm{FLG}_{\mathrm{It}}\left(\mathrm{P}_{\mathrm{F}}\right)=1.0$ for a turbulent surface point and $\mathrm{FLG}_{\mathrm{lt}}\left(\mathrm{P}_{\mathrm{F}}\right)=\gamma\left(\mathrm{P}_{\mathrm{F}}\right)$ for a transitional surface point, where $\gamma\left(\mathrm{P}_{\mathrm{F}}\right)$ is the value of the intermittency function $\gamma$ at the field point $P_{F}$, which takes the value of the intermittency function at the nearest surface point $\mathrm{P}_{\mathrm{S}}{ }^{\text {nt }}\left(\mathrm{P}_{\mathrm{F}}\right)$ within a limiting wall normal distance. According to [6] and [8] the intermittency function can be expressed as

$$
\gamma\left(\mathrm{s}_{\mathrm{q}}\right)=1-\exp \left[-0.412\left(3.36 \frac{\operatorname{MAX}\left(\mathrm{s}_{\mathrm{q}}-\mathrm{s}_{\mathrm{q}, \mathrm{tr}}^{\mathrm{beg}}, 0\right)}{\mathrm{s}_{\mathrm{q}, \mathrm{tr}}-\mathrm{s}_{\mathrm{q}, \mathrm{tr}}^{\text {beg }}}\right)^{2}\right],
$$

where $\mathrm{s}_{\mathrm{q}}$ is the arc length on the airfoil side $\mathrm{q}$ starting at the stagnation point and $\mathrm{s}_{\mathrm{q}, \mathrm{tr}}{ }^{\text {beg }}$ is the location of transition onset. For the determination of the extent of the transitional region, the transition length $\mathrm{l}_{\mathrm{tr}}=\mathrm{s}_{\mathrm{tr}}{ }^{\text {end }}-\mathrm{s}_{\mathrm{tr}}$ beg , the formula from [19],

$$
\operatorname{Re}_{1_{\text {tr }}}=\mathrm{a}\left(\operatorname{Re}_{\delta_{\text {tr }}^{* \text { beg }}}\right)^{\frac{3}{2}},
$$

with $\mathrm{a}=4.6$ for flows with pressure gradient, is applied. For testing purposes, equation (4) with a $=2.3$ is applied, as is recommended in [16] for flows in which transition does not occur before laminar separation. The thickness of the laminar boundary layer $\delta$ is evaluated directly from the Navier-Stokes grid using the 99\%criterion, 


$$
\delta(\mathrm{x})=\left.\mathrm{y}\right|_{\mathrm{U}=0.99 \mathrm{U}_{\mathrm{e}}},
$$

and the compressible Bernoulli equation is used to evaluate the edge velocity $U_{e}$,

$$
\mathrm{U}_{\mathrm{e}}=\sqrt{\mathrm{U}_{\infty}^{2}-\frac{2 \kappa}{\kappa-1} \frac{\mathrm{p}_{\infty}}{\rho_{\infty}}\left[\left(\mathrm{p}_{\mathrm{wall}} / \mathrm{p}_{\infty}\right)^{\frac{\kappa-1}{\kappa}}-1\right]} .
$$

Thus, the displacement thickness $\delta^{*}$ and $\rho_{\mathrm{e}}$ and $\mathrm{U}_{\mathrm{e}}$, the values of the density and the tangential flow velocity at the boundary-layer edge, can be determined. In the case that the value of $U_{e}$ is not reached, the y-location of the maximum value of $\mathrm{U}(\mathrm{y})$ is taken as the value of the boundary-layer thickness.

\section{Results}

The coupled system was applied to the NLF(1)-0416 laminar airfoil [13] with $\mathrm{M}=0.3, \mathrm{Re}=4 \times 10^{6}, \alpha=2.03^{\circ}$ and $\mathrm{N}_{\mathrm{T}}=11$ [17], the limiting $\mathrm{N}$-factor for the TS-database method. In [16] the transition locations $\mathrm{x}^{\mathrm{T}}$ upper $/ \mathrm{c}=0.35$ on the upper side and $\mathrm{x}^{\mathrm{T}}$ lower $/ \mathrm{c}=0.6$ on the lower side are reported for $\mathrm{M}=0.1$, a Mach number which could not be set in the computation because the TAU code version used does not yet provide a preconditioning capability. All computations were started with initial transition locations set at $75 \%$ of chord on upper and lower sides, and the Spalart-Allmaras turbulence model with Edwards modification (SAE) was used for all tests. The transition prediction procedure was run in the $2^{\text {nd }}$ mode.

To ensure the high quality of the laminar boundary-layer data necessary for accurate transition predictions the boundary layer was resolved using 64 cells in the wall normal direction. Within a constant wall distance in the prismatic layer part of the hybrid grid $80 \%$ of the maximum turbulent boundary-layer thickness at the upper side trailing edge are embedded, Fig. 2. Additionally, it seems to be sufficient to use a grid spacing with near wall clustering which is optimised for the resolution of a fully turbulent boundary layer also in the laminar part of the flow. Thus, the very expensive grid adaptation procedure [15], which is normally necessary after each iteration step of the transition locations iteration, could be avoided. The basic parameter settings for the coupling procedure were: a) the overall number of iteration cycles for the RANS computation, cycle $_{\max }=30,000$; b) the cycle interval for the iteration of the transition locations, $\Delta \mathrm{k}_{\mathrm{cyc}}=3,000$; and c) the relaxation factor $\mathrm{f}^{\text {relax }}$ for the underrelaxation of a transition location $\mathrm{x}^{\mathrm{T}}, \mathrm{f}^{\text {relax }}=0.7$, while the underrelaxation formula reads

$$
\mathrm{x}^{* \mathrm{~T}}\left(\mathrm{k}_{\text {cyc }}^{1}\right)=\mathrm{x}^{*^{\mathrm{T}}}\left(\mathrm{k}_{\text {cyc }}^{\mathrm{l}-1}\right)-\mathrm{f}^{\mathrm{relax}}\left[\mathrm{x}^{*^{\mathrm{T}}}\left(\mathrm{k}_{\text {cyc }}^{\mathrm{l}-1}\right)-\mathrm{x}^{\mathrm{T}}\left(\mathrm{k}_{\text {cyc }}^{1}\right)\right] \text {. }
$$

The sensitivity aspects considered are: 1 ) the surface grid point where the turbulence model is activated first, which either can be the nearest point upstream or the nearest point downstream of $x^{* \mathrm{~T}} ; 2$ ) the possible downstream movement of the transition points; 3 ) the consideration of the RANS grid laminar separation points as transition points; 4) the application of point transition vs. the application of transition lengths.

Three cases with different combinations of the sensitivity parameters are presented here. The results of the $1^{\text {st }}$ case, applying point transition, no laminar separation and downstream movement are depicted in Fig. 3. On the left hand 
side, for the nearest point upstream and on the right hand side, for the nearest point downstream, the convergence histories of the transition locations vs. the RANS iteration cycles are shown. In the figures, the transition locations coming directly from the database and the underrelaxed values are depicted. Left: the upper side transition point has overrun the experimental transition point and still has a clear tendency to further move upstream. The lower side transition point has converged to a value which is located $8 \%$ downstream of the experimental value based on the following specification of $\mathrm{b} \%=\left[\left(\mathrm{x}^{\mathrm{T}}\right.\right.$ comp $/ \mathrm{x}^{\mathrm{T}}$ exp $\left.)-1\right] \times 100$. Right: The upper side transition location converges directly to a value which approximates the experimental transition point with an error of $2.86 \%$. The lower side transition location, however shows perturbations which seem to be a more or less periodic back-and-forth oscillation of the transition point from the database.

The results of the $2^{\text {nd }}$ case, applying point transition at the nearest surface grid point downstream of $x^{* T}$ with suppressed downstream movement are depicted in Fig. 4, without laminar separations on the left hand side and with laminar separation on the right hand side. Additionally, the points of laminar separation as they occurred during the computation in RANS grid and the grid points that represent the transition location in the RANS computational grid are depicted in the plots. Left: The back-and-forth oscillation of the transition location on the lower side does not occur, and the transition point converges to a value which is located $7.5 \%$ too far downstream compared to the experimental value. On the upper side, the value of the transition location is the same as in the computations which permitted downstream movement. Right: The lower side transition location is hit almost exactly by a laminar separation point. The convergence of the transition location iteration is accelerated from 3 calls of the transition prediction module to 2 calls.

The results of the $3^{r d}$ case, applying the intermittency function and the transition length model from equation (4) at the nearest surface grid point downstream of $\mathrm{x}^{* \mathrm{~T}}$ with suppressed downstream movement and the use of the RANS grid laminar separation points with $\mathrm{a}=4.6$ on the left hand side and with $\mathrm{a}=2.3$ on the right hand side are depicted in Fig. 5. Left: The results are characterised by strong oscillations of the lower side transition location given by the database. The final lower side transition location, which is based on a laminar separation point, overruns the experimental value slightly and converges to a value which is located about 3.5\% too far upstream. An investigation of the flow field solution [7] reveals a large separation bubble on the upper side starting slightly downstream of the transition point. This was due to a transition length of $13 \%$ of the chord length which is too long - expected is an extent of about $5 \%$ of the chord length - such that the influence of the turbulence model, which should result in an increase of the skin friction, is delayed. Right: The final lower side transition location, which is again based on a laminar separation point, overruns the experimental value slightly and converges to a value which is located about $1.7 \%$ too far upstream. The lower side transition points which are determined by the database after the last laminar separation point was set as a transition point, are located about $7 \%$ downstream of the experimental value. The separation bubble on the upper side 
does not occur [7]. Instead, on the lower side another separation bubble has developed, because now the value of the transition length on the lower side has become significantly larger.

Finally, the effects of the basic coupling parameters $\Delta \mathrm{k}_{\text {cyc }}$ and $\mathrm{f}^{\text {relax }}$ were investigated. $\Delta \mathrm{k}_{\mathrm{cyc}}$ was changed to $\Delta \mathrm{k}_{\mathrm{cyc}}=5,000$ to see if the accuracy of the transition locations might be improved by a longer convergence of the RANS solution between two calls of the transition prediction module. $\mathrm{f}^{\text {relax }}$ was changed to $\mathrm{f}^{\text {relax }}=$ 0.5 to see if a slower convergence of the transition location iteration has an influence on the final values of the transition locations. The tests were carried out only for the settings without transition lengths and without taking into account the laminar separation points. Each of these changes did not influence the final values of the transition locations on upper and lower sides of the airfoil [7].

With respect to the accuracy of the predicted transition locations it seems to be justified to compare the results from a computation at $\mathrm{M}=0.3$ and the experimental values at $\mathrm{M}=0.1$, especially because an incompressible stability analysis using the database method was performed such that the only compressible influences possible may come from the RANS solution. On the other hand, the predicted transition locations are of the same - very good - order of accuracy that were obtained using the DLR block-structured FLOWer code and the transition prediction module applying them to the same test case at $\mathrm{M}=0.1$ [4-5].

\section{Conclusions}

The hybrid TAU code coupled to a transition prediction module was successfully applied to a subsonic airfoil test case automatically taking into account the transition locations predicted by an $\mathrm{e}^{N}$-database method or which were based on laminar separation points determined by the TAU code. The experimentally measured transition locations could be determined with very high accuracy when the nearest surface grid point downstream of the predicted transition location is used as transition point, when a possible downstream movement of the transition points is suppressed and when point transition is applied instead of transitional flow models. A sensitivity study of the parameters of the coupling procedure showed that the accuracy of the converged transition locations can be highly improved and the convergence of transition location iteration itself can be significantly accelerated when the laminar separation points which occur in the RANS computational grid during the transient phase of the computation are immediately used as transition locations. In contrast to these encouraging results, the application of the intermittency function based on algebraic transition length models for the modelling of transitional flow regions led to large separated regions directly downstream of the transition point. These separated flow regions are contrary to experimental findings on the one hand, and lead to strong disturbances in the convergence of the RANS computation on the other. Therefore, at present the application of these models in the TAU code together with the SAE turbulence model can not be recommended. Further investigations are necessary here to clarify why the transitional flow models showed such an unexpected behaviour. 


\section{Acknowledgements}

This work has been carried out within the HiAer Project (High Level Modelling of High Lift Aerodynamics) and documented in detail in [7]. The HiAer project was a collaboration between DLR, ONERA, KTH, HUT, TUB, Alenia, Airbus Deutschland, QinetiQ and FOI. The project was managed by FOI and was partly funded by the European Union (Project Ref: G4RD-CT-2001-00448).

\section{References}

[1] FLOWer - Installation and User Handbook, Release 116, Doc.Nr. MEGAFLOW-1001, Institut für Entwurfsaerodynamik, Deutsches Zentrum für Luft- und Raumfahrt e.V., 2000

[2] Horton, H. P., Stock, H. W., "Computation of Compressible, Laminar Boundary Layers on Swept, Tapered Wings", Journal of Aircraft, Vol.32, No. 6, 1995, pp.1402-1405

[3] Kroll, N., Rossow, C.-C., Schwamborn, D., Becker, K., Heller, G., "MEGAFLOW - A Numerical Flow Simulation Tool For Transport Aircraft Design", ICAS Congress 2002, Toronto (can), 09.-13.09.2002, ICAS, CD-Rom, pp. 1.105.1-1.105.20, 2002

[4] Krumbein, A., Stock, H. W., "Laminar-turbulent Transition Modeling in Navier-Stokes Solvers using Engineering Methods", Barcelona, ECCOMAS 2000-CD-Rom Proceedings

[5] Krumbein, A., "Coupling of the DLR Navier-Stokes Solver FLOWer with an eN-Database Method for laminar-turbulent Transition Prediction on Airfoils", Notes on Numerical Fluid Mechanics · Volume77, pp. 92-99, Germany 2000, Springer Verlag, 2002

[6] Krumbein, A., "Transitional Flow Modeling and Application to High-Lift Multi-Element Airfoil Configurations", Journal of Aircraft, Vol. 40, 2003, pp. 786-794

[7] Krumbein, A. et al., HiAer Deliverable D3.1-2: "Implementation of transition/turbulence models", Technical Report, April 2004

[8] Krumbein, A., "Automatic Transition Prediction and Application to High-Lift Multi-Element Airfoil Configurations", AIAA-2004-2543, July 2004 (sub. to Journal of Aircraft)

[9] Nebel, C., "Transitionsberechnung an einem 3D Rumpfkörper", Institutsbericht 2003/1, Institut für Strömungsmechanik, Technische Universität Braunschweig, 2003

[10] Nebel, C., Radespiel, R., Wolf, T., "Transition Prediction for 3D Flows Using a ReynoldsAveraged Navier-Stokes Code and N-Factor Methods", AIAA-2003-3593

[11] Radespiel, R., Graage, K., Brodersen, O., "Transition Predictions Using Reynolds-Averaged Navier-Stokes and Linear Stability Analysis Methods", AIAA Paper 91-1641, 1991

[12] Smith, A. M. O., Gamberoni, N., "Transition, Pressure Gradient and Stability Theory", Douglas Aircraft Company, Long Beach, Calif. Rep. ES 26388, 1956

[13] Somers, D. A., "Design and Experimental Results for a Natural-Laminar Flow Airfoil for General Aviation Applications", NASA Technical Paper 1861, Scientific and Technical Information Branch, 1981

[14] Stock, H. W., Degenhardt, E., "A simplified $\mathrm{e}^{\mathrm{N}}$ method for transition prediction in twodimensional, incompressible boundary layers", Zeitung für Flugwissenschaft und Weltraumforschung, Vol.13, 1989, pp.16-30

[15] Stock, H. W., Haase, W., "A Feasibility Study of $\mathrm{e}^{\mathrm{N}}$ Transition Prediction in Navier-Stokes Methods for Airfoils", AIAA Journal, Vol.37, no. 10, 1999, pp. 1187-1196

[16] Stock, H. W., Haase, W., "Navier-Stokes Airfoil Computations with $\mathrm{e}^{\mathrm{N}}$ Transition Prediction Including Transitional Flow Regions", AIAA Jour., Vol.38, no.11, 2000, pp.2059-2066

[17] Stock, H. W., "Airfoil Validation Using Coupled Navier-Stokes and $\mathrm{e}^{\mathrm{N}}$ Transition Prediction Methods", Journal of Aircraft, Vol.39, No. 1, 2002, pp.51-58

[18] van Ingen, J. L., "A suggested Semi-Empirical Method for the Calculation of the Boundary Layer Transition Region", University of Delft, Dept. of Aerospace Engineering, Delft, The Netherlands, Rep. VTH-74, 1956

[19] Walker, G. J., "Transitional Flow on Axial Turbomachine Blading", AIAA Journal, Vol.27, No. 5,1989 , pp. 595-602 


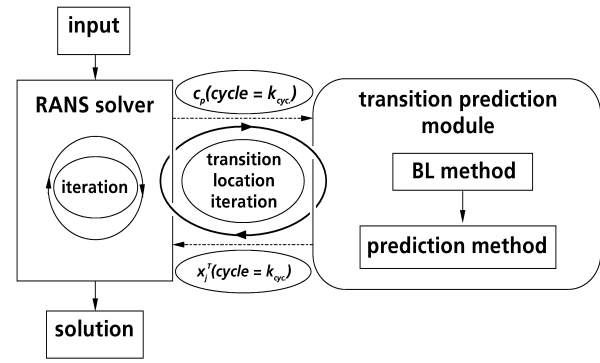

Fig. 1 Transition prediction coupling structure

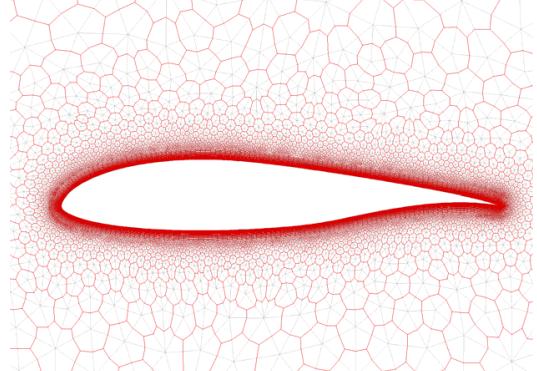

Fig. 2 Dual grid and hybrid primary grid
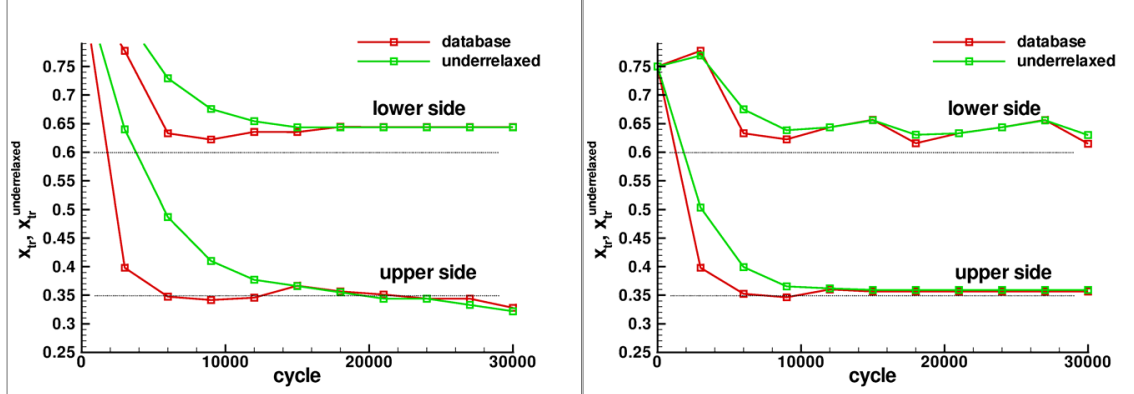

Fig. 3 Convergence of the transition locations, no laminar separation, no transition lengths, downstream movement, left: nearest point upstream, right: nearest point downstream
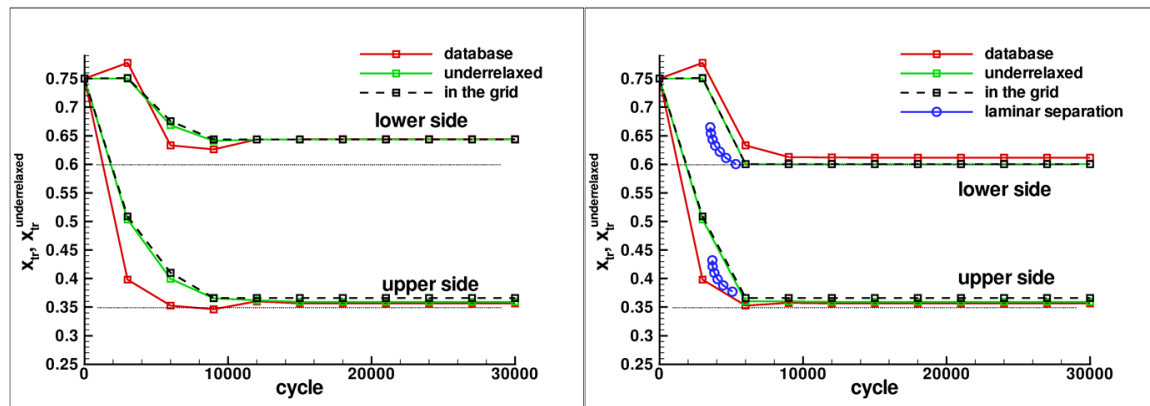

Fig. 4 Convergence of the transition locations, nearest point downstream, no transition lengths, no downstream movement, left: no laminar separation, right: with laminar separation
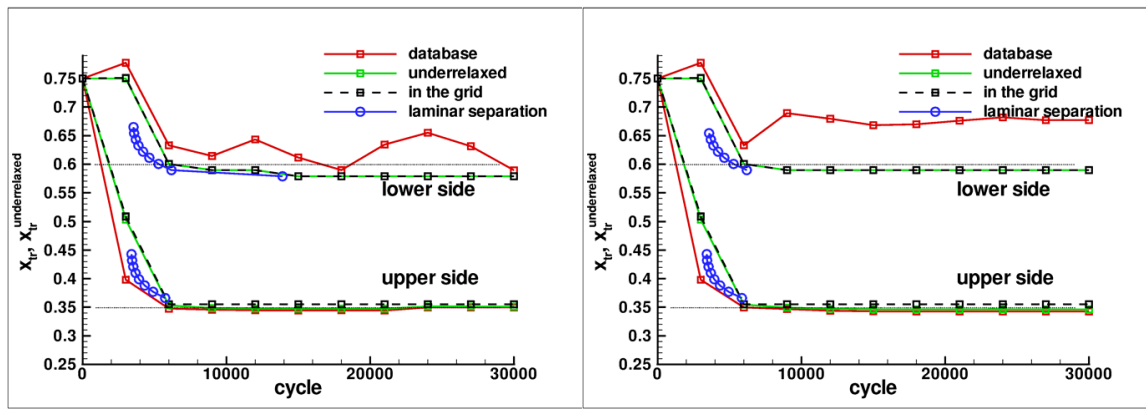

Fig. 5 Convergence of the transition locations, nearest point downstream, with laminar separation, no downstream movement, transition lengths, left: $\mathrm{a}=4.6$, right: $\mathrm{a}=2.3$ 